\title{
Emotional and Cognitive Responses of Children Attending Summer Camps in Occupied Palestine: A Pilot Study
}

Amira Oudeh ${ }^{1}$, Tahmina Nizam ${ }^{1}$, Ian Barron ${ }^{2 *}$, Ghassan Abdallah ${ }^{\mathbf{3}}$ and Peter Willatts ${ }^{1}$

'School of Psychology, University of Dundee, United Kingdom

${ }^{2}$ School of Education, Social Work and Community Education, University of Dundee, UK

${ }^{3}$ Centre for Applied Research in Education, Ramallah, Occupied Palestine, Palestine

\author{
Abstract \\ Background: The current study sought to address the lack of evaluation for summer camps which seek to offer \\ emotional and cognitive support for children in occupied Palestine.
}

Objective: To assess children's emotional and cognitive response to summer camps, children who attended camps were compared to those who did not attend on a standardised measure of emotion and cognition within situations of adversity.

Methods: A mixed methods quasi-experimental post-test only design was used.Children, aged 8-10 years, who attended a summer camp $(n=62)$, were compared to those who had not attended a camp $(n=22)$ on the Child Post-traumatic Cognitions Inventoryand on a qualitative summer experience questionnaire. Camp workers $(n=16)$ also completed a summer experiences questionnaire for comparison of perceptions betweenworkers and children. Statistical analysis involved t-test and ANOVA for within and between group differences and a six-step quasi-qualitative analysis was used to assess summer experience questionnaire responses.

Results: Unexpectedly,children who had attended a summer camp presented higher levels of traumatic cognition; however, they also reported more hopefulness for the future than children who did not attend a camp. It is uncertain if camp attendees are a self-selecting group because of higher levels of traumatic exposure and/or cognitions or because summer camps may have unintended negative consequences.

Conclusion: The current study, with its unexpected results, emphasises the importance of embedding evaluation into summer camp delivery. More robust experimental designs, however, are necessary in order to be confident that the outcomes are related to program rather than contextual factors. A range of potential summer camp and environmental influencing factors on outcomes were identified for future research.

Keywords: Trauma; Emotion; Cognition; Summer camps

\section{Introduction}

Children in Occupied Palestine have experienced the effects of military occupation since birth, and some might argue through the perinatal period [1]. In a recent study in the West Bank, children on average experienced eleven different types of war event, e.g. witnessing a member of the family killed, or experiencing being detained and beaten by military personnel [2]. The consequences of cumulative violence in Palestinian children, including intergenerational trauma, are becoming increasingly apparent. A wide range of resultant developmental trauma symptoms have been identified including post-traumatic stress, traumatic grief, depression and school under achievement [2]; dissociation and self-harm and drug misuse [3]. In contrast to the high incidence of military/domestic violence and resultant symptoms, services to support children in the occupied Palestinian territories are scarce [4]. Further, given the stressed economic context, the likelihood of the development of anything but small-scale therapeutic services seems unlikely.

As a viable alternative, community-oriented approaches have emerged as a potential solution to address the substantial numbers of children experiencing adversity. Within a context of limited resources and expertise along with a communal culture of family, community and education, summer camps have evolved in an attempt to address children's emotional and cognitive responses to violence [5]. In contrast to the delivery of therapy, which requires specialised knowledge and skills, summer camps can be delivered by a range of community personnel; these have included teachers, community workers and local and international volunteers.

Summer camps in occupied Palestine vary considerably. Some are run by the National Committee for Summer Camps (NCSC), while others are organised by non-governmental organisations (NGOs). Camps differ in size, facilities, activities and employee numbers. Depending on the religious orientation of the community, camps can be single sex or mixed. Most are stratified by age groups covering a wide age range 5-16years. While the duration of camps varies, most tend to cover a 2 week period. A range of activities can be delivered including play, cultural activities, sports, community service, education, and environmental awareness [6]. The settings in which camps operate also vary with regard to the different types and levels of violence experienced by communities [4]. Despite these differences, both nonNCSC and NCSC summer camps have similar aims for children, i.e. to build children's emotional, cognitive and social capacity to cope with life under occupation.

\section{Limited evaluation to date}

Although there has been recent growth in the number of summer camps in occupied Palestine, there has been little systematic evaluation

*Corresponding author: lan Barron, School of Education, Social Work and Community Education, University of Dundee, Nethergate, Dundee DD1 4HN, United Kingdom, Tel: +44 1382 381479; E-mail: i.g.z.barron@dundee.ac.uk

Received October 24, 2014; Accepted December 12, 2014; Published December 19, 2014

Citation: Oudeh A, Nizam T, Barron I, Abdallah G, Willatts P (2014) Emotional and Cognitive Responses of Children Attending Summer Camps in Occupied Palestine: A Pilot Study. J Psychol Abnorm Child 4: 136. doi:10.4172/2329-9525.1000136

Copyright: ( 2014 Oudeh A, et al. This is an open-access article distributed under the terms of the Creative Commons Attribution License, which permits unrestricted use, distribution, and reproduction in any medium, provided the original author and source are credited. 
of the benefits of summer camps for children [5]. Where there have been studies, these have tended to be qualitative in nature, lacking in a systematic approach to research design and analysis and as a result, typically remain as grey literature. Internationally, the situation is little better. Part of the difficulty has been a confusion of definition regarding what is and what is not a summer camp. Definitions for example, appear to differ depending on the cultural context. In the US, summer camps often seek to augment educational attainment for children, whereas in the UK camps can be play and social skills oriented. Within occupied Palestine however, the focus has been on limiting children's military contact and the building of children's resilience. This is the focus of the current study.

Where there has been evaluation, the impact of summer camps is inconclusive. Tol and colleagues conducted a meta-analysis of mental health efficacy studies in countries with humanitarian crises from 20072010 [7]. Of the 753 studies analysed, only three evaluated the impact of recreational, cultural and non-formal activities on children's well-being. Of these, only two studies showed gains in child well-being. In a postconflict northern Uganda study of the impact of psychosocial structured activities (PSSA) on trauma symptoms, Ager et al. [8] found the mental health of children aged 7-12 years $(n=203)$ improved naturally over 12 months. PSSA involved 15 one-hour sessions over 2 weeks and included a wide range of activities such as drama, music, art, coping strategies and safety discussions. PSSA also included a parent component with regular parental meetings to discuss child and community needs. Although many factors could have influenced outcomes over the year, the authors still suggested that PSSA can have a beneficial impact on children's emotional, cognitive and social development.

The second study within the meta-analysis which focused on recreational activities was within occupied Palestine. Loughry et al [5] worked with two NGOs to conduct a year-long comparison-group design study on non-formal structured activities with children $(n=400)$ aged 6 to 17 years in Gaza and the West Bank. Children experienced cultural, recreation and sport activities during week-long summer camps, with parents encouraged to participate or observe activities. Unlike Ager et al. [8], Loughry and colleagues sought to increase children's resilience by establishing a sense of normality through local and global community engagement, e.g. by focusing on safe play areas and connecting to children in other countries via the internet. Using the Child Behaviour Checklist, the authors found a reduction in behavioural difficulties and an increased perception of parental support. A range of other factors however, such as school counselling, was acknowledged as potentially influencing outcomes.

Within the small number of summer camp outcome studies, there has been little systematic assessment and analysis of children's subjective experience. Studies of children's response to trauma recovery programmes in occupied Palestine however, have started to address this issue. Barron and Abdallah [9] utilised a focus group approach to discover Gazan children's experience of the Healing Trauma Combating Hatred programme. The study utilised a quasi-qualitative approach to thematic analysis. The authors concluded children were able to share their emotional experience prior to and following the programme and identify which parts of the programme were more effective. The same authors, utilising an interview approach with 133 children in the West Bank highlighted Palestinian children's ability to share their thoughts and experiences of the Teaching Recovery Techniques programme. Despite a culture of non-disclosure young people were able to share their worries and fears, identify programme limitations and suggest ideas for programme improvement [2]. In relation to summer camp evaluation, it is therefore suggested, Palestinian children's perspectives may be a rich untapped source of information for evaluative researchers and summer camp planners.

Psycho-social summer camp studies in occupied Palestine have almost exclusively used qualitative approaches. Only Loughry et al. [5] administered a standardised measure. As the current study sought to assess changes in children's emotional capacity and cognitions in situations of adversity, the child's Post-Traumatic Cognitions Inventory (cPTCI) [10] was identified as a measure with known validity and reliability [11-13]. In addition, this measure was readily available to use, as the Arabic version had recently been translated by the Centre for Applied Research in Education, Ramallah (CARE). The cPTCI was originally designed to identify the emotional and cognitive consequences for children who experience a traumatic or adverse event. The cPTCI consists of two sub-scales related to viewing the self as incompetent and the world as a scary place. The cPTCI is short, simple to administer and suitable for a wide age range in situations of adversity [14]. The cPTCI has however, yet to be validated for children in contexts of communal cumulative violence and no studies to date have evaluated the Arabic version. Questions are based upon the adult version, the Post-Traumatic Cognitions Inventory [15] with ageinappropriate questions excluded. Although this measure has validity, there is still uncertainty about how similar adult and child emotional and cognitive experiences are under adversity.

\section{The present study}

The effectiveness of summer camps in occupied Palestine has been taken on trust rather than based on empirical evidence. There has been a lack of published studies, systematic research designs, standardised measures and a limited approach to qualitative analysis. The current study sought to address these issues. A mixed methods quasiexperimental post-test only design was used to explore the impact of summer camps on the emotional and cognitive capacity of children in a context of military occupation. Scores on the on the Arabic version of the cPTCI from children aged 8-10 years who attended a summer camp were compared to those of children who did not attend a camp. In addition, children's subjective experience of summer time was assessed by a student summer experience questionnaire. Camp workers also completed a summer experiences questionnaire for comparison of their perceptions with those of the children.

\section{Methods}

\section{Participants}

Participants were children attending summer camps during the month of July within the three districts of Ramallah, Jerusalem and Jericho. These areas were identified by CARE because of the high levels of military violence, occupation restrictions (travel and water) and unemployment [15]. This study included summer camps governed by the Ministry of Sports and Youth and run on NCSC guidelines for structure, staffing and activities. The camps covered four different geographical locations in the West Bank: Kufr Ni'meh, Qatanna, Qalandia (refugee camp) and Jericho. Four out of six summer camps in these areas volunteered to participate in the project. The two camps that declined participation were not sufficiently staffed to release workers. With the exception of a girls' camp in Qatanna, all camps included boys and girls aged 5-16 years. All camps ran for a fortnight.

Participants of both genders were selected through convenience sampling, using the following criteria: eight to ten years of age; present at the time of questionnaire completion; and their camp worker was available to be present during questionnaire administration. This 
age group fitted well with the cPTCI. Out of a possible 85 children, 68 children ( 25 males, 43 females; mean age 9.22 years) volunteered to participate. In total, 16 out of 19 adults completed the summer experience questionnaire. The reason for the three adults' nonparticipation was lack of availability at the time of questionnaire administration due to camp work demands.

An equivalent number of children $(n=22)$, aged eight to ten years, who did not attend a summer camp were selected from two of the four areas. This restricted sampling occurred because only the summer camps in Qatanna and Qalandia were able to select children from the appropriate age range who did not attend summer camp.

In the camp in Kufr Ni'meh, the participants were 15 children (6 males, 9 females: mean age 9.73 years) in the summer camp group (SC), and four camp workers. In the Jericho camp, there were 11 children (6 males, 5 females: mean age 8.64 years) in the SC group, and five 5 camp workers. In the Qatanna camp, there were 9 girls (mean age 9.0 years) in the SC group, 11 children (4 males, 7 females: mean age 8.91 years) in the non-summer camp group (NSC), and three camp workers. In the Qalandia camp, there were 11 children ( 2 males, 9 females: mean age 9.64 years) in the SC group, 11 children in the NSC group (7 males, 4 females: mean age of 9.18 years), and four camp workers.

\section{Measures}

Child post-traumatic cognitions inventory: Children from SC and NSC groups completed the Arabic version of cPTCI. This 25-item self-report measure consists of two subsets "Disturbing and permanent change" (Permanent Change) and "Feeble person in a scary world" (Scary World) as well as having a total score (Total). The Scary World subscale has 12 statements with maximum score of 48 and Permanent Change subscale has 13 statements with maximum scores of 52 . These are rated on a Likert-type scale with responses of "Don't agree at all" = 1; "Don't agree a bit" = 2; "Agree a bit" = 3; and "Agree a lot" $=4$. The maximum score for Total equals 100 .

Summer experience questionnaire: The summer experience questionnaire for children was translated from English into Arabic by an experienced Palestinian translator (Educational Psychologist by profession) and checked by one of the researchers, a fluent ArabEnglish speaker, for accuracy. Six questions explored different aspects of children's experience during the 2 week summer camp experience. Themes included: support received; what children enjoyed the most; how their experiences affected their thoughts and feelings; as well as anything that made children feel unhappy. As noted previously, the latter addressed a gap in the research where evaluation often omits the possibility of negative programme impact. The camp workers were asked the same questions but to think of the child's experience. For example, "what impact do you think summer camp has on children's thoughts and feelings?"

\section{Procedure}

Ethical approval was obtained through the University of Dundee
Research Ethics Committee. Active informed consent and assent was required from parents, camp workers and children respectively. Posttest measures were administered by two researchers present, a week following the end of the summer camp. Administration of the cPTCI, and immediately following, the summer experience questionnaire, took place on a whole group basis for summer camp and non-summer camp children, within each of the camps in classroom-like environments. The summer experience questionnaire was administered to camp workers individually by the two researchers.

\section{Analyses}

Descriptive statistics initially explored differences between groups and areas for cPTCI total scores, the Scary World component and Permanent Change component scores. Independent t-tests were used to analyse Total, Permanent Change and Scary World score differences between summer camp and non-summer camp groups. Two-way ANOVA was used to analyse camp differences (Qalandia vs. Qatanna) and group type (summer camp vs. non-summer camp) for Total, Permanent Change and Scary World scores. ANCOVAs were then conducted to investigate whether age or sex had a confounding effect on cPTCI scores. Means and standard deviations in the results section are presented as (mean $\pm \mathrm{SD})$.

Children and camp workers responses were analysed using an adapted six-step thematic analysis [16] requiring familiarisation with data; generating initial codes; searching for themes; rank ordering initial codes; reviewing and naming themes; and drafting a report. Themes were coded using numbers, with frequency of themes noted for each test group. Themes were also ranked according to relevance to research question and occurrence in groups. Themes and codes are communicated as ratios of statements to codes. To assess interrater reliability, a researcher (educational psychologist) also analysed the qualitative questionnaires. Cohen's $d$ was calculated for child and worker themes.

\section{Results}

\section{cPTCI scores}

Due to the failure to complete the cPTCI because of camp nonattendance, one summer camp male from Kufr Ni'meh; one summer camp female and two summer camp males from Qatanna and two summer camp males from Jericho were omitted from the statistical analysis.Mean and standard deviations for total and subcomponent scores of cPTCI using area and group are shown in Table 1.

A significant effect was found for summer camp compared to non-summer camp attendance Total scores $(\mathrm{t}(62)=3.60, \mathrm{p}=0.001$, $\left.\eta_{\mathrm{p}}{ }^{2}=0.42\right)$, i.e. children in summer camps $(48.62 \pm 9.34)$, scored higher emotional and cognitive disturbance scores, than children not attending summer camp $(39.68+9.61)$. Similarly, a significant effect of group type $\left(\mathrm{t}(32.36)=2.89, \mathrm{p}=0.007, \eta_{\mathrm{p}}{ }^{2}=0.45\right)$ was found for Scary World, i.e. children in summer camps scored higher $(24.62 \pm 4.13)$, than children

\begin{tabular}{|c|c|c|c|c|c|c|c|c|}
\hline \multirow[t]{2}{*}{ Area } & \multirow[t]{2}{*}{ Group } & \multirow[t]{2}{*}{$\mathbf{N}$} & \multicolumn{2}{|c|}{ Total } & \multicolumn{2}{|c|}{ Scary World } & \multicolumn{2}{|c|}{ Permanent Change } \\
\hline & & & M & SD & M & SD & M & SD \\
\hline Kafr Ni'ma & Summer camp & 14 & $(43.29$ & $\pm 7.75)$ & $(22.14$ & $\pm 3.51)$ & $(21.14$ & $\pm 5.10)$ \\
\hline Jericho & Summer camp & 9 & $(56.56$ & $\pm 6.39)$ & (27.22 & $\pm 3.60)$ & (29.33 & $\pm 4.80)$ \\
\hline \multirow{2}{*}{ Qatanna } & Summer camp & 8 & $(57.50$ & $\pm 4.69)$ & $(27.12$ & $\pm 2.85)$ & $(30.38$ & $\pm 3.42)$ \\
\hline & Non summer camp & 11 & (33.73 & $\pm 8.95)$ & (18.91 & $\pm 4.49)$ & (14.82 & $\pm 3.84)$ \\
\hline \multirow{2}{*}{ Qalandia Camp } & Summer camp & 11 & $(42.45$ & $\pm 5.52)$ & $(23.82$ & $\pm 4.17)$ & (18.64 & $\pm 1.96)$ \\
\hline & Non summer camp & 11 & $(45.64$ & $\pm 6.01)$ & $(22.27$ & $\pm 4.77)$ & (23.36 & $\pm 4.11)$ \\
\hline
\end{tabular}

Table 1: Total, Scary World and Permanent Change score means and SDs by area and group. 
not attending summer camp (20.59 \pm 5.82$)$ and for Permanent Change $\left(\mathrm{t}(62)=3.02, \mathrm{p}=0.004, \eta_{\mathrm{p}}{ }^{2}=0.36\right)$, i.e. again children in summer camps scored higher $(24.00 \pm 6.35)$, than children not attending summer camp $(19.09 \pm 5.85)$. No significant effects were found for age $[F(1,61)=$ $0.075, \mathrm{p}=0.785]$ or gender $[\mathrm{F}(1,61)=0.255, \mathrm{p}=0.615]$.

Since results could be biased by political/geographical areas, further analyses were carried out on areas with attendees and nonattendees (Qatanna and Qalandia Camp). A significant difference was found between Total for summer camp group in Qatanna and Qalandia Camp (t $\left.(28)=5.83, \mathrm{p}<0.001, \eta_{\mathrm{p}}{ }^{2}=0.74\right)$. Participants in Qatanna, (57.50 \pm 4.69$)$, scored significantly higher than those in Qalandia Camp $(42.45 \pm 5.52)$. Likewise there was significant difference between Total for non-summer camp groups in Qatanna and Qalandia Camp ( $\mathrm{t}(20)$ $=-3.67, \mathrm{p}=0.002, \eta_{\mathrm{p}}^{2}=0.63$ ).

To test whether subcomponents of cPTCI contributed equally to Total further analyses were carried out. A 2 (area: Qalandia Camp, Qatanna) x2 (group type: summer camp, non-summer camp) betweensubjects ANOVA was tested on Scary World. There was a significant effect of group type $\left(F(1,37)=10.08, p=0.003, \eta_{p}^{2}=0.21\right)$ and interaction $\left(\mathrm{F}(1,37)=4.71, \mathrm{p}=0.37, \eta_{\mathrm{p}}{ }^{2}=0.11\right)$. Nonetheless, area was not significant $(\mathrm{F}(1,37)<0.001, \mathrm{p}=0.985)$. A 2 (area: Qalandia Camp, Qatanna) x2 (group type: summer camp, non-summer camp) betweensubjects ANOVA was conducted using Permanent Change, found a very significant effect of group type $\left(\mathrm{F}(1,37)=24.99, \mathrm{p}<0.001, \eta_{\mathrm{p}}{ }^{2}=\right.$ $0.40)$ and interaction $\left(F(1,37)=87.69, \mathrm{p}<0.001, \eta_{\mathrm{p}}{ }^{2}=0.70\right)$. However again area, showed no significant effects $(\mathrm{F}(1,37)=2.17, \mathrm{p}=0.149)$.

To explore group effects in these areas in more detail, $\mathrm{t}$-tests were carried out on Total, -SW and -PC in Qatanna. There was a significant effect of group type on Total $\left(\mathrm{t}(17)=6.83, \mathrm{p}<0.001, \mathrm{\eta}_{\mathrm{p}}{ }^{2}=0.86\right)$. Children in the summer camp group $(57.60 \pm 4.69)$ had higher scores than the non-summer camp group (33.73 \pm 8.95$)$. There was a significant effect of group type on Scary World $\left(\mathrm{t}(17)=3.34, \mathrm{p}=0.004, \eta_{\mathrm{p}}{ }^{2}=0.63\right)$. Children in the summer camp group $(27.12 \pm 2.85)$, scored higher than the nonsummer camp group $(18.91 \pm 6.49)$. There was a significant effect of group type on Permanent Change $\left(\mathrm{t}(17)=9.11, \mathrm{p}<0.001, \eta_{\mathrm{p}}{ }^{2}=0.91\right)$. In comparison to children in the non-summer camp group (14.82 \pm 3.84$)$, summer camp children had higher scores (30.38 \pm 3.42$)$.

Likewise t-tests were carried out on Total, Scary World and Permanent in Qalandia to test if there were significant differences between group types. There was no significant effect of group type on Total and Scary World $(\mathrm{p}>0.05)$. However there was a significant effect on Permanent Change ( $\mathrm{t}(20)=-3.45, \mathrm{p}=0.003, \eta_{\mathrm{p}}{ }^{2}=0.61$ ). Children attending summer camp (18.64 \pm 1.96$)$, had lower scores than children not attending $(23.36 \pm 4.11)$.

\section{Summer experience questionnaire responses}

Children attending summer camps had six key themes directly related to emotional and cognitive development. One third of participants referred to 'emotional satisfaction', i.e. theme coded 3 with 14 statements (3:14). One participant said "the camp gave me happiness". Almost a quarter mentioned 'positive cognitive development' (6:9), e.g. "It made me think well and more accurately". Under a quarter wrote about 'child development' (5:6), e.g. "Improving self-confidence", and 'support' (4:4), e.g. "The help provided for us by teachers and supervisors". Over a third wrote about 'education' (1:17) through "learning about my town and my country". The most common theme was 'social skills' (2:40). One child wrote of how they "used to sit alone but now I like to sit with a group". The main themes and subthemes, based on responses from children in Qantanna and Qalandia can be seen in figure 1 .

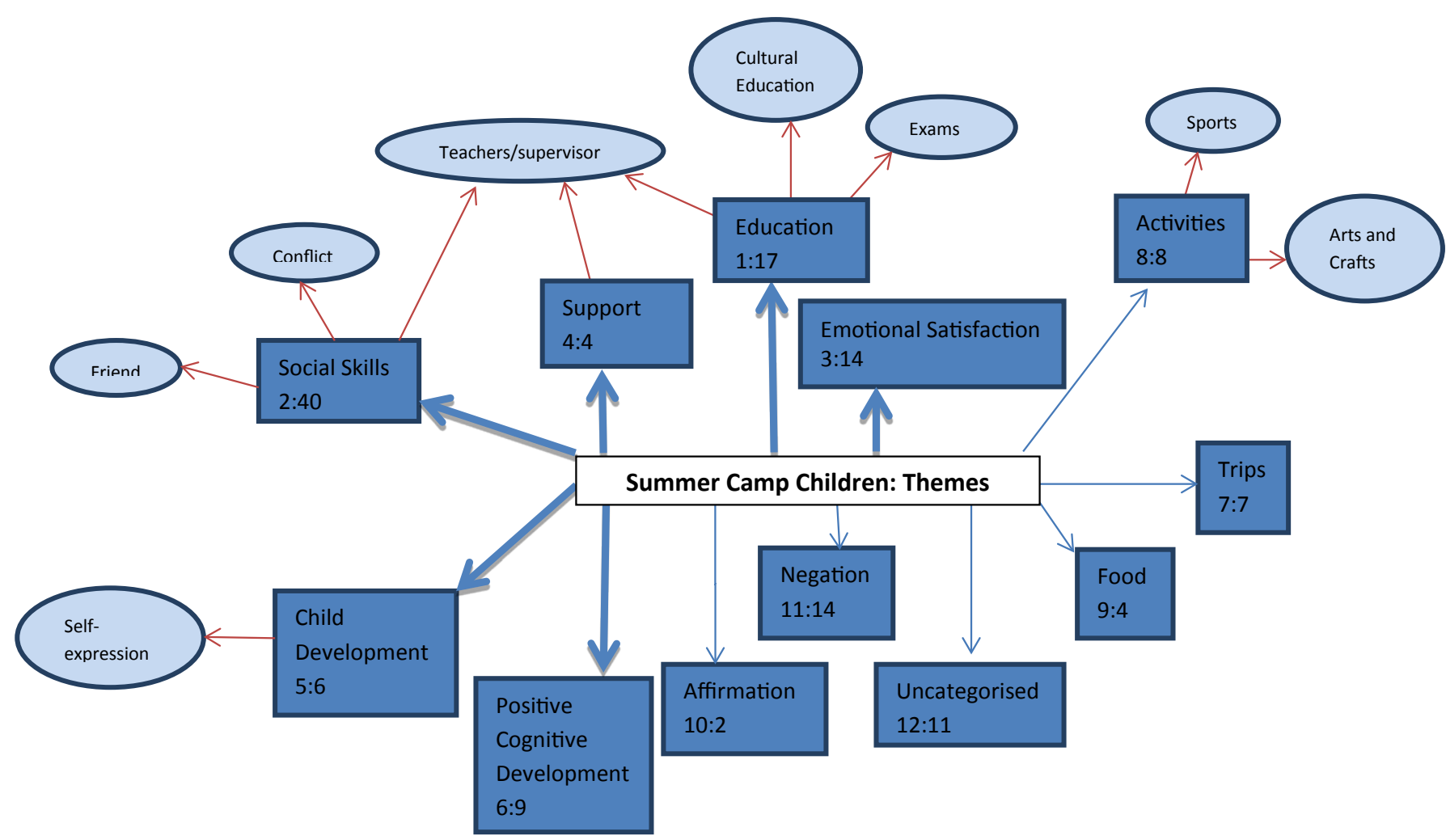

Figure 1: Thematic Analysis of Summer Camp in Qatanna and Qalandia. 


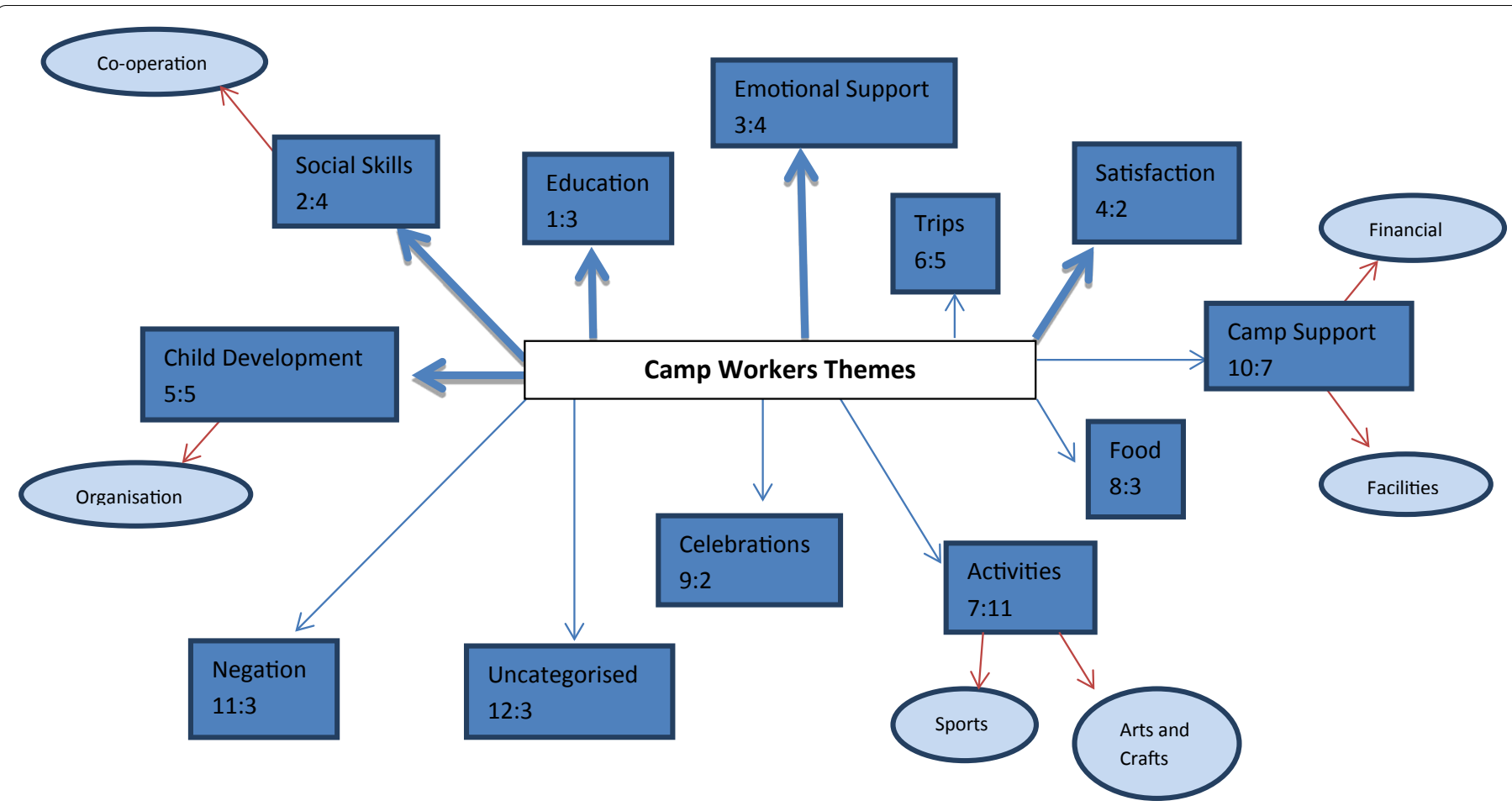

Figure 2: Thematic Analysis of Camp Workers in Qatanna and Qalandia.

Six other common themes were unrelated to the research question. This included 'activities' (8:8) like "Sport as it makes you stronger"; 'trips' (7:7) including "having more distant trips"; and food (9:4). There was an 'uncategorised' group (12:11), e.g. "In a better a fashion". Thus 126 responses were placed in 12 categories.

For camp workers, nine themes were identified from 35 statements. The main themes and sub-themes, based on responses from workers in Qantanna and Qalandia can be seen in Figure 2. Six of the themes were directly related to the research question. In order of descending frequency, these included 'camp support' (10:7), e.g. "no place for setting up camp which made some children think that a camp is the same as a school"; 'child development' (5:5), e.g. "breaking the barrier of fear or shyness"; 'emotional support' (3:4), e.g. "nothing to make the child feel unhappy"; 'social skills' (2:4), e.g. "gaining new friends"; 'education' (1:3), e.g. "educates through play and leisure" and 'satisfaction with the camp experience' (4:2), e.g. "happiness and joy".

The most prevalent theme, unrelated to the research question, was 'activities' (7:11), e.g. "drawing, colouring". Other themes include 'trips' (6:5); 'food' (8:3), e.g. "food buffet can be provided"; 'celebrations' (9:2), e.g. "parties" and 'uncategorised' responses (12:3), e.g. "I don't think so". Themes and sub-themes for camp workers' responses can be found in Figure 2.

Inter-rater reliability of the themes for young people and workers was high, $d=0.89$ and $d=0.91$ respectively. This was partly due to the structured and focused nature of the questions and the small sample size. An additional theme of 'potential negative consequences' was identified for both children who attended summer camps and their workers.

\section{Discussion}

Child mental health is undoubtedly a complex part of cognitive neuroscience, driven by different feedback processes underlying the genetic, social or environmental system [17]. For children living under occupation, the current study found conflicting evidence for the impact of summer camps on children's emotional and cognitive responses. For refugee children in a summer camp in Qatanna, attendance may have led to increased hopefulness for the future and a greater understanding of the possibilities of change compared to children who did not attend camp. The research paradigm however leaves such a conclusion speculative until pre/post-test randomised designs are utilised. In contrast to potential positive gains, children who attended summer camp in Qalandia, were found to have higher levels of emotional and cognitive distress compared to non-attendees at post-test. It is possible therefore, as with other psycho-social interventions, that summer camps are not neutral in their effect, i.e. camps may have the potential to increase children's distress. For example, two of the camps were highly structured in activity, whereas the other camps encouraged more self-directed activity; some camps were located next to military barriers while others were further away from these overt signs of conflict and it may be that differing levels of camp staff traumatisation could have had an impact on staff capacity to mediate children's trauma response. Alternatively, the higher levels of emotional and cognitive distress found in the summer camp children could have resulted from selection bias. Summer camp attendees for example, may have been a self-selecting group because of their higher symptoms of distress. Similarly, summer camps may have prioritised children who reported most distress. Camp workers comments suggest the latter. The interactions of all potential influencing factors however, require further research. This would be a significant development in the field as the unintended negative consequences of interventions are often neglected in evaluative studies [18].

Regardless of whether the findings suggest gains or negative consequences, indications are that summer camp structures and activities appear insufficient to address children's emotional and cognitive distress. This is perhaps not surprising as current research 
into trauma recovery suggests trauma-specific interventions, rather than psycho-social activities are necessary for trauma resolution [19]. Summer camps in Palestine provide only the latter. While there may be the possibility of gains through support, these gains are unlikely to be maintained in the long term without trauma-specific interventions [20].

Despite the lack of evidence to suggest summer camps impact on children's symptoms of trauma, the findings do suggest some camps may be able to impact on children's cognitive appraisal of their experience of adversity, e.g. increased hope for the future. Given the levels of child depression in the West Bank [2], such a potential outcome is not insignificant. Future studies therefore need to explore the differing structures and activities of camps in order to assess which features of camps are most likely to lead to positive cognitive appraisal.

Although not to significant levels, children's distress differed across camps geographically. In comparing Qatanna and Qalandia, the trend appears to be that children from communities of greater adversity experience higher symptoms levels, a finding supported by other studies [21]. Given this was not a significant finding, the relationship between adversity and symptom severity may be complex with a range of factors contributing to children's differential symptom levels. Camps, for example, are located within communities of differing economic deprivation levels [22] as well as different forms of political and military control. Within the current study, children in communities of greater poverty tended to view the world as a more fearful place. On the other hand, differing community security arrangements in which camps operate may also have had a differential impact on children's emotional and cognitive responses [23]. Qalandia for example, is in a zone controlled by the Palestinian National Authority, whereas Qatanna is controlled by both the Palestinian National Authority and Israeli military and is split across two militarised zones. Children in the latter community then, have to cope with a community partly controlled by a perceived oppressor state with increased military contact through checkpoints, searches and incursions. All these experiences can lead to increased stressors [3].

Cultural factors in Palestinian society may also have influenced differential levels of distress for children across communities. Some communities, for example, are more fundamentally religious than others which in turn impacts on children's self-expression and potentially children's capacity for healing [4]. In the current study children in such areas tended to display higher symptom levels. Gender is another significant factor in Palestinian society with distinct male and female roles in more traditional communities. It is surprising then, that no gender difference was found in the current study. In contrast, previous studies suggest girls tend to show higher levels of distress [2]. Finally, some communities have become more westernised than others as a consequence of a culture of created dependency [24]. Currently there is no evidence to suggest children in camps in these communities cope any better or worse than more traditional communities.

As a comparison to the standardised measure, children's subjective experience indicated similar expressions of emotional satisfaction with their summer experience whether attending a camp or otherwise. Children who attended camps however, tended to be more specific in the naming emotional gains, e.g. a growth in self-confidence and happiness. Further camp attendees identified a wider of developmental benefits, i.e., increased ability to think; learning about country and culture, improved social skills and inclusion in groups as well as an increased sense of being supported. Within a context of occupation these are potentially significant developmental gains. Interestingly, such findings are congruent with studies which explore the impact of summer camp attendance within non-war contexts [25]. Whether children would have made measurable gains on standardised measures in these factors is unknown, however, this finding, supported by summer camp workers perceptions, does affirm that summer camps through their milieu and psycho-social activities may have a role in growing children positive experiences and potentially increasing their resilience. Taken together with the $\mathrm{CPTCI}$ scores indications are that some summer camps may impact positively on children's sense of wellbeing and cognitive appraisal of situations of adversity. An appropriate experimental research design however is required to address this issue.

As with children's responses, summer camp workers perceived camps as providing a range of benefits, i.e. an educative experience, emotional support and a social context for inclusion and social skill development. Camp workers however were more cautious about the impact of camps with one voicing concern that camps held in schools may not give children a sufficiently different experience from being at school. The impact of this issue requires further research.

\section{Limitations}

There are a range of methodological limitations associated with post-test only designs. Without pre-test measures there is no way to know whether children's scores have changed between before and after camp attendance, i.e., whether children self-selected (or adults selected them) to attend because of higher levels of traumatic cognitions or whether camps do harm. Likewise a lack of randomisation to summer camp and non-summer camp introduces uncertain biases about the nature of the samples. In this study the sample sizes were also small raising questions about the representativeness of participants to the wider population. Further this research was conducted with young people of a specific age range. In contrast, Kilmer and Gil-Rivas [26] argue that adversity affects children and youth at different ages because their emotional and cognitive capacities make them comprehend and internalise the event differently. It may be the age-range in the current study was too narrow to identify any age difference. Assessment wise, only one standardised measure was used. Without a comparison measure, it is difficult to assess the reliability of findings. In addition, assessment only explored short term gains for children. Finally, with the semi-qualitative analysis, the frequency of any theme did not denote the importance of the theme, merely the number of times raised.

\section{Conclusions}

The current study highlights the complex and uncertain relationship between childhood adversity, and geographical, political and cultural factors involved in summer camp effectiveness. Children attending summer camps unexpectedly presented higher levels of traumatic cognition yet reported more hopefulness for the future. It is uncertain if camp attendees are a self-selecting group because of higher levels of traumatic cognitions or because summer camps may do harm. Many questions about the nature of children's response to adversity and the importance of adequately assessing participant characteristics prior to camp attendance have been raised. Indications are that summer camps may have the potential to both support on the one hand and lead to unintended consequences on the other. Given the limitations of the research design, the range of potential influencing factors and the uncertain meaning of the findings, it is premature to make recommendations for practice. What can be suggested is that summer camps need to engage in more adequate assessment of the needs of the children and the communities they come from in embedding evaluation into their own practice. Indications are that trauma specific programmes 
Citation: Oudeh A, Nizam T, Barron I, Abdallah G, Willatts P (2014) Emotional and Cognitive Responses of Children Attending Summer Camps in Occupied Palestine: A Pilot Study. J Psychol Abnorm Child 4: 136. doi:10.4172/2329-9525.1000136

Page 7 of 7

may be necessary if children are to experience transformational healing from trauma. This issue was out with the scope of the current study.

\section{Recommendations for Research}

Future studies should utilise experimental research designs. A randomised control trial of summer camp effectiveness would be a substantial development in research design in this field. Studies need to utilise a range of standardised measures to assess different aspects of summer camp psychosocial goals, e.g. anxiety, depression and resiliency. The potential of summer camps to create unintended negative consequences also needs to be assessed. Longitudinal studies would be useful in identifying any longer term impact of camp attendance.

\section{References}

1. Svraka E, Loga S (2006) War psychotrauma of pregnant women as prenatal factor of cerebral palsy. Medical Archives 60: 376-378.

2. Barron I, Abdullah G, Smith P (2012) Randomised control trial of a CBT recovery program in Palestinian schools. Journal of Loss and Trauma: International Perspectives on Stress and Coping 18: 306-321.

3. Punamäki R, Qouta S, El Sarraj E (2001) Resiliency factors predicting psychological adjustment after political violence among Palestinian children. Int J of Dev Behav 25: 256-267.

4. Barron I, Abdallah G (2013) Intergenerational trauma in the occupied Palestinian Territories Submitted to the $\mathrm{J}$ Child and Adol Trauma. In Press.

5. Loughry M, Ager A, Flouri E, Khamis V, Afana A, et al. (2006) The impact of structured activities among Palestinian children in a time of conflict. J Child Psychol Psychiatry 47: 1211-1218.

6. UNICEF (2002) UNICEF Support to Summer Camps in the Occupied Palestinian Territories.

7. Tol WA, Barbui C, Galappatti A, Silove D, Betancourt TS, et al. (2011) Mental health and psychosocial support in humanitarian settings: linking practice and research. Lancet 378: 1581-1591.

8. Ager A, Akesson B, Stark L, Flouri E, Okot B, et al. (2011) The impact of the school-based psychosocial structured activities (PSSA) program on conflictaffected children in Northern Uganda. J Child Psychol Psychiatry 52: 11241133

9. Barron I, Abdullah G (2012) Evaluation of the efficacy of a group-based trauma recovery program in Gaza: Students' subjective experience. Journal of Trauma and Loss: International Perspectives on Stress and Coping 17: 187-199.

10. Meiser-Stedman R, Smith P, Yule W, Dalgleish T (2003) How l've been thinking and feeling since the frightening event.
11. Meiser-Stedman R, Smith P, Bryant R, Salmon K, Yule W, et al. (2009) Development and validation of the child post-traumatic cognitions inventory (cPTCl). J Child Psychol Psychiatry 50: 432-440.

12. Bryant RA, Salmon K, Sinclair E, Davidson P (2007) A prospective study of appraisals in childhood posttraumatic stress disorder. Behav Res Ther 45 2502-2507.

13. Ellis A, Nixon R, Williamson P (1999) The effects of social support and negative appraisals on acute stress symptoms and depression in children and adolescents. Brit J Clin Psychol 48: 347-361.

14. Perrin S, Meiser-Stedman R, Smith, P (2005) The Children's Revised Impact of Event Scale (CRIES): Validity as a screening instrument for PTSD. Behav Cogn Psychoth 33: 487-498.

15. Foa E, Ehlers A, Clark A, Tolin D, Orsillo S (1999) The posttraumatic cognitions inventory (PTCl): development and validation. Psychological Assessment 11 : 303-314.

16. Braun V, Clarke V (2006) Using thematic analysis in psychology. Qual Res Psychol 3: 77-101.

17. Fareed M, Afzal M (2014) Estimating the inbreeding depression on cognitive behavior: a population based study of child cohort. PLoS ONE 9: e109585.

18. Barron I, Topping K (2010) School-based child sexual abuse prevention programs: Implications for practitioners. American Professional Society on the Abuse of Children 22: 11-19.

19. Rodenburg R, Benjamin A, Roos C, Meijer A, Stams G (2009) Efficacy of EMDR in children: A meta-analysis. Clin Psychol Rev 29: 599-606.

20. Becker J, Greenwald R, Mitchell C (2011) Trauma-informed treatment for disenfranchised urban children and youth: An open trial. Child Adolesc Social Work J 28: 257-272.

21. Mousa H (2011) 5 Injured in Demonstrations at Qalandia Checkpoint. Retrieved 4th April, 2012

22. UNICEF (2010) The Situation of Palestinian Children in the Occupied Palestinian Territory, Jordan, Syria and Lebanon. UNICEF, Jordan.

23. Giacaman R, Khatib R, Shabaneh L, Ramlawi A, Sabri B, et al. (2009) Health status and health services in the occupied Palestinian territory. Lancet 373 837-849.

24. Portland Trust (2011) Reducing Aid Dependency in the Palestinian Territory Economic feature, Portland Trust, Ramallah.

25. Epstein I Stinson J, Stevens B (2005) The effects of camp on health-related quality of life in children with chronic illnesses: a review of the literature. Pediatr Oncol Nurs 22: 89-103

26. Kilmer RP, Gil-Rivas V (2010) Exploring posttraumatic growth in children impacted by Hurricane Katrina: Correlates of the phenomenon and developmental considerations. Child Dev 81: 1211-1227. 